\title{
Application of Brain Neural Network in Personalized English Education System
}

\author{
https://doi.org/10.3991/ijet.v13i10.9488 \\ Yang Songlin, Zhang Min $\left({ }^{凶}\right)$ \\ Beihua University, Jilin, China \\ Mariazhang97@126.com
}

\begin{abstract}
This paper aims to rectify the various deficiencies of the traditional teaching models. To this end, the memorization of English words was cited as a case to simulate the English word learning system, and a brain neural network (BNN) was built to analyse the learner's forgetting curve. Then, the learning content was customized according to the curve, aiming to achieve the goal of the personalized education system (PES). Finally, the proposed model was validated through an experiment, which reveals that the forgetting curve generated by the BNN adapt to the learner's memory pattern better than the Ebbinghaus memory curve. Compared with the traditional education models, the new model includes the profiles and natures of the learner, making the teaching more fruitful and scientific. Thanks to the BNN and computer technology, the PES in the proposed system is highly flexible and cost-effective, shedding new light on the popularization of personalized education.
\end{abstract}

Keywords-personalized education system (PES); brain neural network (BNN); English education; forgetting curve

\section{Introduction}

The information era has witnessed the springing-up of new knowledge and the growth of knowledge-based economy. However, the traditional education models, despite their popularity and scalability, cannot adapt to the disparity in learners' intellectual levels, cognitive competence and curriculums [1-2]. Against this backdrop, more and more attention has been paid to improve the traditional education models. One of the trendiest new education models is personalized education system (PES) [3].

The PES refers to a set of teaching programs tailored according to the potentials, education backgrounds, qualities and occupations of individual learners. In other words, this education model teaches students in accordance of their aptitude. The existing studies on the PES concentrate on the competency-based teaching system through reform in the context of traditional education [4-5]. Nonetheless, the PES is too costly to be promoted widely. The cost of the PES mainly include the heavy inputs of manpower, material and financial resources. 
With the development of computer technology, brain science, cognitive science and interdisciplinary studies, many scholars are probing into the PES from the perspective of the brain neural network (BNN) [6]. A number of artificial neural network models have been developed, mimicking BNNs [7-9]. These network models can automatically analyse the learners' profiles from their feedback data, and sheds new light on reducing the education cost in the PES.

In this paper, the memorization of English words is cited as a case to simulate the English word learning system, and a BNN is built to analyse the learner's forgetting curve. Then, the learning content was customized according to the curve, aiming to achieve the goal of the PES.

\section{PES framework based on neural network}

\subsection{PES model and workflow}

The model and workflow were established for the PES based on neural networks. As shown in Figure 1, the whole system consists of such four modules as learner, the PES platform, the prior knowledge, and the neural network.

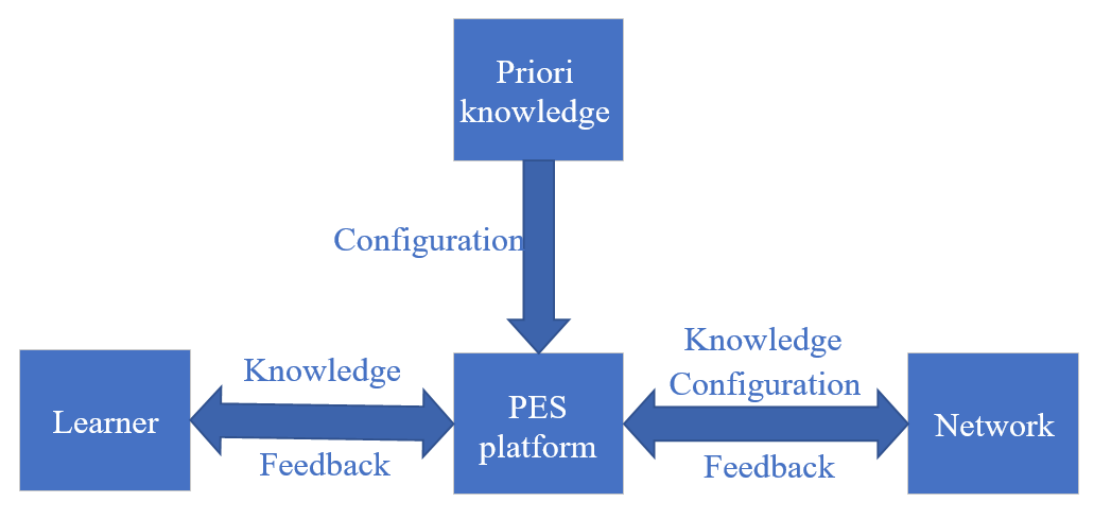

Fig. 1. PES model and workflow

Specifically, the learner refers to those who acquire knowledge using the PES. The learner module mainly gives a feedback to the knowledge provided by the education platform. The feedback may fall into the categories of very familiar, familiar, unfamiliar, fuzzy, etc. The PES platform, located between the learner and the neural network, is responsible for transmitting knowledge, learner's feedback and configuration (which is based on the prior knowledge) between the other three modules. The prior knowledge includes learning time, learner's profile, coverage bias of the education system, etc. The neural network is a prediction module of the PES model. In addition to training, this module can make a prediction according to the knowledge from the PES platform and the feedbacks from the learner. The prediction may facilitate the management of the teaching contents. 
Upon the reception of a learning request from the learner:

$\mathrm{C}=$ Priori. generate config ()

$=$ Network. Predict $(K, C)$

$\mathrm{K}^{\prime}=\left\{\mathrm{k} \mid \mathrm{k} \in \mathrm{K}^{\wedge}\right.$ need_to_learn $\left.(\mathrm{b})^{\wedge}(\mathrm{k}, \mathrm{b}) \in(\mathrm{K} . \mathrm{B})\right\}$

return $\mathrm{K}^{\prime}$

upon the reception of a feedback $(k, b)$ from the learner:

$\mathrm{C}=$ Knowledge.generatre_config ()

Network.train $(k, C, b)$

Algorithm 1 Operation of the PES model

The system operation is defined by Algorithm 1, where lines $1 \sim 5$ specify the reaction of the PES model to a learning request from the learner. First, the system reads the configuration from the prior knowledge on the response to the request from the other learners, which may involve time and learner's profile (line 2). Then, the configuration and knowledge base should be transmitted to the neural network to get a feedback from the network about each entry of knowledge in the knowledge base (line 3). After that, the system needs to filter out the knowledge based on the pre-set judgment function of the feedback, and determine whether the learner needs to learn certain knowledge (line 4). Finally, the filtered knowledge is returned to the learner (line 5). Lines $6 \sim 8$ of the Algorithm is the workflow of the PES after the learner feeds back certain knowledge. This time, the system still needs to read a configuration from the prior knowledge (line 7), and then transmit the knowledge, configuration, and feedback to the neural network for training (line 8).

\subsection{Flexibility and low cost of the PES}

The traditional education systems often require manual filtering of knowledge based on learner's feedback and of the teaching contents based on the prior knowledge. For example, the teaching progress should be adjusted according to the known learning capacity of the learner. Coupled with the disparity between learners, the growing demand of the learner for learning contents will inevitably raise a huge demand for manpower.

In the proposed PES, the learner's feedback is process by the neural network and the computer in a highly automatic manner. The demand for manpower remains at the same level of complexity, thanks to the expansion of the knowledge base. Thus, the teaching cost is reduced greatly in the proposed model.

Moreover, the proposed model is loosely coupled. In traditional education systems, the teacher must perceive the constant changes in the number and learning features of the learners and make corresponding adjustments. In our model, it is only necessary to modify the prior knowledge base due to the loose coupling. This also contribute to the reduction in the teaching cost invoked by the changes of the learner.

To sum up, the BNN-based PES model is very flexible and cost-saving. In the next section, a BNN is set up to analyse the forgetting curve of the learner during the memorization of English words. This curve enables the customization of learning coverage for the learner. The author conducted an experiment to compare the curve against the 
universal Ebbinghaus memory curve, aiming to verify the teaching effect of the proposed model.

\section{Case study}

\subsection{PES for the memorization of English words}

The abstract elements of the PES framework were instantized for the memorization of English words (Table 1). Considering the important effect of duration on learning, the configuration was instantiated as a timestamp; the knowledge base was instantiated as a set of English words to be memorized by the learner; the feedback is instantiated into such two types as known and unknown, to represent the response of the learner upon receiving the knowledge from the base.

Table 1. English word memorization system

\begin{tabular}{|c|c|}
\hline Abstract unit & Instance unit \\
\hline Configuration & Timestamp \\
\hline Knowledge base & Set of English words \\
\hline Feedback & "Known" or "unknown" \\
\hline
\end{tabular}

Upon receiving a learner's request to learn/preview English words, the English word memorization system (EWMS) will transfer the current time and word library selected by the learner into the neural network, which will then return a set of labels corresponding to the word library. These labels indicate which words need to be learned and which do not. Subsequently, the EWMS returns a collection of words that need to be learned to the learner.

When the learner gives a feedback on the familiarity with a certain word (presented as "known" or "unknown"), the EWMS will transmit the current time, the word, and the feedback all at once to the neural network.

In addition, the neural network was instantiated as a multilayer perceptron (MLP) [8-10]. The details on the MLP are provided in the next subsection.

\subsection{The MLP}

With a forward structure, the MLP is an artificial neural network (ANN) that maps a set of input vectors to a set of output vectors. It can be regarded as a directed graph of multiple node layers, each of which is fully connected to the next layer. Except for the input nodes, each node is a neuron or processing unit with a nonlinear activation function. The MLP is often trained by the supervised learning method called the backpropagation algorithm [11].

The MLP can be abstracted into a training function and a prediction function. The training function trains the stateful MLP using the samples and labels as inputs, while 
the prediction function makes a prediction based on the samples and outputs a label as the result.

In the EWMS, the timestamp and English words are adopted as samples, and the learner's feedback as a label to train the MLP. The timestamp and English words were also taken as samples to make the prediction about whether the learning content is suitable for the learner.

It is worth mentioning that the training and prediction of the MLP can be carried out alternatively, forming a loop of training-prediction-training. In the EWMS, there is another loop: learning-feedback-relearning. These two loops are isomorphic, which ensures that the MLP can satisfy the demands of the EWMS.

In the EWMS, the learner's feedback on the learned knowledge is used as a part of the training sample, and, in the subsequent relearning, the knowledge to be learned is given by the prediction. Of course, the prediction result is influenced by the feedback from the previous learning. In this way, there will be a feedback loop across the entire system (Figure 2).

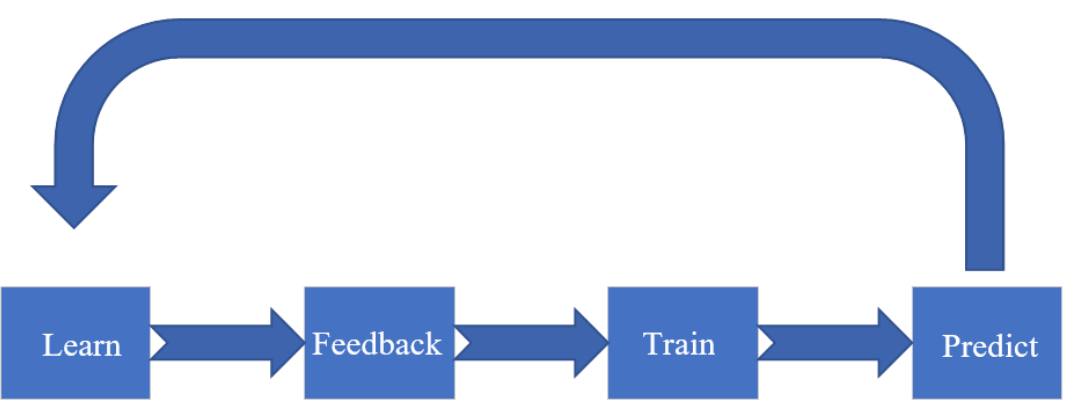

Fig. 2. Feedback loop in the EWMS

\subsection{Experiment}

This subsection aims to verify the learning effect of the EWMS through experiment. Before the experiment, 20 subjects were selected and randomly divided into an experimental group and a control group. Each subject was required to learn 100 English words through a three-step learning process: initial acquaintance, review and testing.

The initial acquaintance means the learner acquires a preliminary understanding of the English words; the review means the learner gives a feedback on the learning effect, i.e. whether he/she know the English words to be learned (If the words are unknown, a relearning should be arranged); the testing means the learner receives a test on whether he/she has mastered the English words to be learned. No learning is involved in the testing process.

Both groups of subjects underwent the initial acquaintance step at the start of the experiment, reviewed the learned words after $20 \mathrm{~min}, 1 \mathrm{~h}, 12 \mathrm{~h}, 1 \mathrm{~d}$ and $2 \mathrm{~d}$, respectively, and received the testing $7 \mathrm{~d}$ later. The first step covers all English words to be learned, while the second step covers 50 words. 
Only the experimental group learned the English words through the proposed EWMS. During the review, half of the English words were selected by the PLS. Once the review phase ends, the feedback from the subjects is transferred to the system as the training data for the MLP. By contrast, the control group learned all 100 English words and reviewed them at the said time points. Note that half of the words for each review were chosen randomly.

Figure 3 presents the mean accuracy of each group concerning the memorization of the English words to be learned.

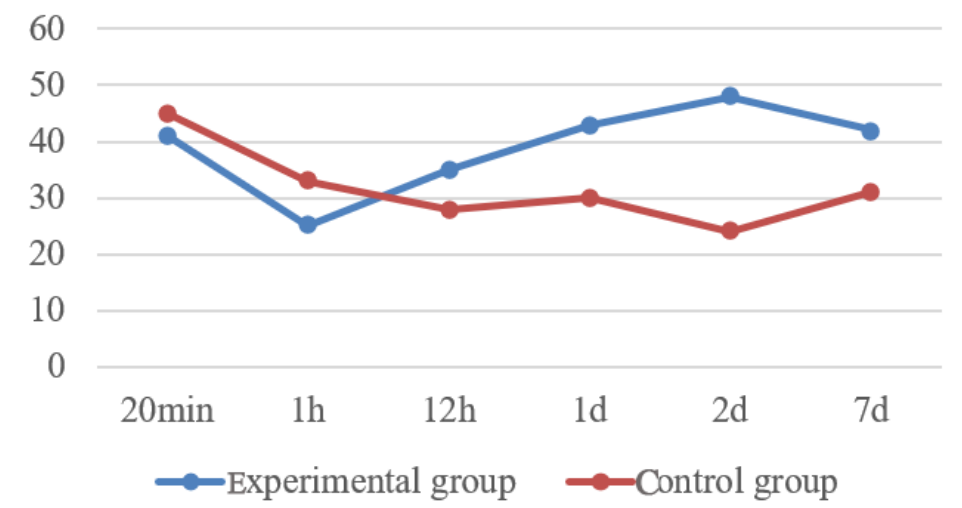

Fig. 3. Mean accuracy of each group concerning the memorization of the English words to be learned

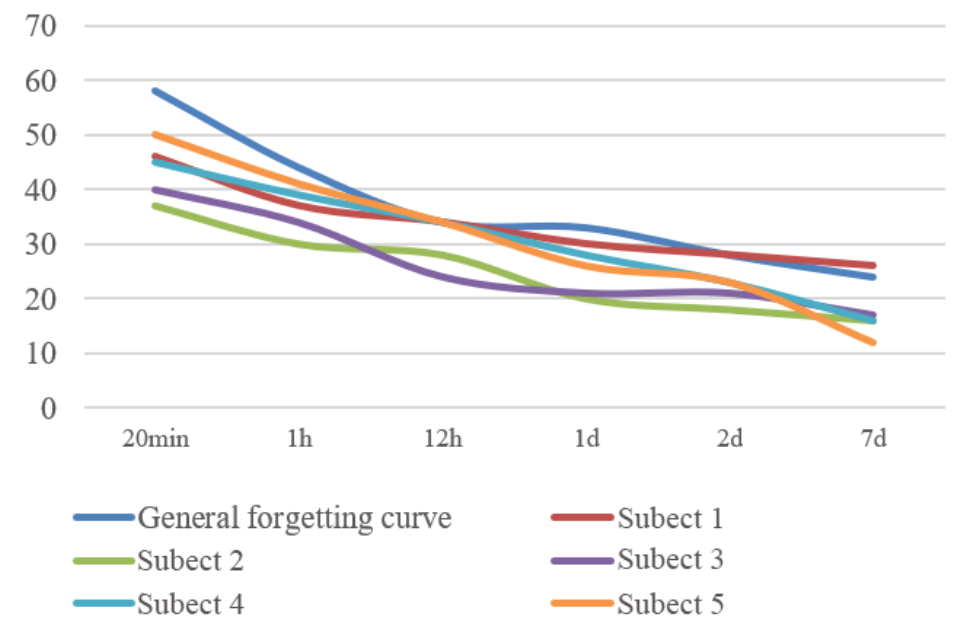

Fig. 4. Comparison between the forgetting curves of different subjects and the general forgetting curves 
As shown in Figure 3, there was no marked difference between the two groups in the mean accuracy at the initial stage. This means the two groups have no gap in review accuracy. However, the gap widened as the review phase continued to increase. In the final testing phase, the experimental group achieved a better mean accuracy than the control group. For better understanding of the effect of the EWMS, the forgetting curves of some subjects in the experimental group were plotted below (Figure 4).

It can be seen that none of the forgetting curves of the subjects was the same as the general Ebbinghaus memory curve [12], which is widely adopted in the traditional systems for the memorization of English words. Unlike the traditional systems, the EWMS tailors the learning schedules for the learners according to their own profiles. Through the experiment, it is clear that the BNN-based forgetting curves adapt to the learner's memory pattern better than the traditional Ebbinghaus memory curve. Using these curves, it is possible to improve the effect of the PES and ensure the scientificness of the teaching activities.

\section{Conclusions}

The models and concepts of education are research hotspots in the academia. More and more scholars are aware of the deficiencies of the traditional teaching models. In light of the above, this paper puts forward a systematic framework that integrates the BNN into the PES, and proves the effectiveness of the framework through an experiment on the memorization of English words. Compared with the traditional education models, the new model includes the profiles and natures of the learner, making the teaching more fruitful and scientific. Thanks to the BNN and computer technology, the PES in the proposed system is highly flexible and cost-effective, shedding new light on the popularization of personalized education.

\section{$5 \quad$ Acknowledgment}

This paper is made possible thanks to the "Research on the Top Design of the Humanistic Literacy Education of BTI Adapted to the Socialist Culture Construction", 2017 Jilin Provincial Education Science Planning Project (Grant No.: GH170056) and the "Research on the Mode of Combining the Excellent Traditional Chinese Culture and the Teaching of BTI Based on the Ancient Chinese Fictions and Their English Translations", 2017 Education and Teaching Reform Research Project, Beihua University.

\section{$6 \quad$ References}

[1] Dewey, J. (1938). Traditional vs. progressive education. Dewey, Experience and edu-cation, 17-23.

[2] Gardner, H. (1989). To open minds: Chinese clues to the dilemma of contemporary education. Basic Books. 
[3] Pykett, J. (2009). Personalization and de-schooling: Uncommon trajectories in con-temporary education policy. Critical Social Policy, 29(3), 374-397. https://doi.org/10.1177/0261 $\underline{018309105176}$

[4] Cutler, T., Waine, B., Brehony, K. (2007). A new epoch of individualization? Prob-lems https://doi.org/10.1111/j.1467-9299.2007.00672.x

[5] Fok, (2004). Apple WP, and Horace HS Ip. Personalized education: an exploratory study of learning pedagogies in relation to personalization technologies International Conference on Web-Based Learning. Springer, Berlin, Heidelberg, 407-415.

[6] Schalkoff, R. J. (1997). Artificial neural networks. New York: McGraw-Hill.

[7] Krizhevsky, A., Sutskever, I., Hinton, G. E. (2012). Imagenet classification with deep convolutional neural networks Advances in neural information processing systems, 1097-1105.

[8] Grossberg, S. (2013). Recurrent neural networks. Scholarpedia, 8(2), 1888. https://doi.org/10.4249/scholarpedia.1888

[9] Gardner, M. W., Dorling, S. R. (1998). Artificial neural networks (the multilayer per-ceptron) - a review of applications in the atmospheric sciences. Atmospheric envi-ronment, 32(14-15), 2627-2636. https://doi.org/10.1016/S1352-2310(97)00447-0

[10] Rumelhart, D. E., Hinton, G. E., Williams, R. J. (1986). Learning representations by backpropagating errors, nature, 323(6088), 533. https://doi.org/10.1038/323533a0

[11] Anderson, J. R., Schooler, L. J. (1991). Reflections of the environment in memory. Psychological science, 2(6), 396-408. https://doi.org/10.1111/j.1467-9280.1991.tb00174.x

\section{$7 \quad$ Authors}

Songlin Yang, author, lecturer of English Department, Foreign Languages College, Beihua University, Jilin 132013, China. Specialized in translation studies and practice, Yang takes the lead in several teaching reform and social science projects. He has published a series of articles and books on translation and English education.

Min Zhang, corresponding author, English teacher, Foreign Languages College, Beihua University, Jilin 132013, China. Zhang takes the lead in several projects of teaching innovation. She has published several articles on Chinese core magazines and a number of books on English education.

Article submitted 12 July 2018. Resubmitted 29 July 2018. Final acceptance 13 August 2018. Final version published as submitted by the authors. 